\title{
La medida de la calidad educativa: acerca de los rankings universitarios
}

\author{
Educational Quality Measurement: On University Rankings
}

\author{
Alejandra Montané, José Beltrán y António Teodoro²
}

\section{Resumen}

En el presente texto se revisa desde una perspectiva crítica la construcción de sistemas transnacionales de evaluación de la calidad a través de los rankings internacionales. En la primera parte, reflexionamos sobre el concepto de calidad y presentamos diferentes tipos de rankings: los denominados League Tables (ARWU, THE, QS) y los centrados en resultados de investigación (SCImago Institutions Ranking). Una vez conocidas las características y posibles utilidades de los mismos, analizamos dialógicamente una serie de riesgos a tener en cuenta para acabar incidiendo de nuevo en el modelo de calidad que proponen y sus posibles efectos.

\section{Palabras clave}

Calidad, evaluación, educación superior, rankings.

\section{Abstract}

The present text assumes a critical stance to examine the design of transnational systems of quality assessment through international rankings. First, different categories of ranking procedures are presented, namely, those known as League Tables (ARWU, THE, QS); and those centred on research outcomes (SCImago, Institutions Ranking). Next, after introducing their potential uses, we dialogically set these types of rankings against a series of risks worth considering. And last, we conclude by offering a reflection on the quality model reflected by each of them, and on their overall effects.

\section{Key words}

Quality, evaluation, higher education, rankings.

1 Los autores pertenecen al grupo de Clacso Universidades y politicas de educación superior. Este artículo se enmarca en el proyecto Erasmus + de la Unión Europea TO-INN. From Tradition to Innovation in Teacher Training Institutions. Referencia: 573685-EPP-1-2016-1-ES-EPPKA2-CBHE-JP.

2 Alejandra Montané López, Universitat de Barcelona, smontane@ub.edu; José Beltrán Llavador, Universitat de València, jose.beltran@uv.es; António Neves Duarte Teodoro, Centro de Estudos Interdisciplinares em Educação e Desenvolvimento (CeiED), Universidade Lusófona de Humanidades e Tecnologías, Lisboa, teodoro.antonio@gmail.com 


\section{Introducción}

Una determinada concepción de la evaluación de la calidad de la educación superior ampara conceptual e ideológicamente el auge de la aparición de los rankings mundiales de universidades que son resultado de la globalización, como refieren Marope, Wells, y Hazelkorn (2013:8), y una señal inequívoca de que las universidades viven realmente «en una época de mediciones y comparaciones». Los rankings, cuyo origen es deportivo (Barsky, 2014), se interpretan como un reflejo de la calidad absoluta de las instituciones de educación superior, o por lo menos, un modo creíble de cuantificarla.

Partiremos del supuesto de que la propia noción de calidad es polisémica, compleja, no estática y no se puede entender más que de manera relacional, esto es, poniéndola en juego dialécticamente con otras dimensiones como las relativas a la excelencia, gobernanza o equidad, y a las que hemos prestado atención en ocasiones anteriores (Beltrán y Montané, 2015; Beltrán et al., 2016; Montané et al., 2017). Consideramos que el fundamento de cada instrumento de evaluación está en la definición que se adopte de calidad, incluso Pérez-Esparrells y López-García (2009) argumentan que es erróneo considerar que los rankings son un mecanismo idóneo para medir la calidad de la educación superior.

Esta suerte de razón instrumental asocia el fenómeno de los rankings, por una parte, con la internacionalización de la educación superior dirigida hacia «economías intensivas en conocimiento» y a la búsqueda global de talentos (Hazelkorn, 2015), por otra parte, remite a las tendencias de mercantilización de la educación universitaria alentando una «carrera por el prestigio» -término aún más discutible- destinada a incidir en la elección de universidades por parte de los estudiantes (Hazelkorn, 2015).

Este contexto globalizador y de mercado se hace patente a través del interés de muchos Estados por contar con universidades de élite atractivas para los investigadores de alto nivel y los inversores. Todo ello con el objetivo de convertirse en motores de la generación y difusión de conocimiento a nivel mundial consolidando el modelo de las world class universities - las que están en puestos relevantes de los rankings- basadas en un discurso ideológico y político comprometido con la adecuación de los sistemas de educación superior de todo el mundo a los requisitos del modelo de producción de los mercados (Santos et al., 2016). En este sentido, los gobiernos de algunos países han fomentado la internacionalización y han financiado la excelencia reformando el modelo de gobernanza de las universidades (Wang, Cheng y Liu, 2012). Así, compartimos la hipótesis de la «gobernanza epistémica», según la cual asistimos a una gobernanza que opera sobre las concepciones acerca de la realidad social de los sujetos (Alasuutari y Qadir, 2016:633-652) $\mathrm{y}$ «afecta a las relaciones de poder en los modos de crear, estructurar, coordinar y aplicar el conocimiento» (Campbell y Carayannis, 2013) sostenida a través de una nueva imagen de la geopolítica internacional del conocimiento (Bruner y Uribe, 2007).

Las temáticas relacionadas con la evaluación de la calidad universitaria son múltiples, complejas e interrelacionadas y aunque en el presente texto incidimos en aspectos generales de los rankings y sus riesgos desde una perspectiva sociológica, otros interesantes estudios como el de Barsky (2014) trata los procesos por los cuales las revistas académicas internacionales (dominantemente en inglés) determinan el sistema de organización de la información científica, por ejemplo, con la creación del Institute for Scientific Information (ISI) que mantiene una íntima relación, y es un aliado indispensable, en la elaboración actual de los rankings.

En nuestro texto presentamos, en primer lugar, una aproximación crítica al uso de la noción de calidad, una descripción conceptual y metodológica de los principales rankings seguida de una reflexión en torno a los principales riesgos que éstos conllevan. 


\section{Crítica de la calidad pura}

Los escenarios recientes de la calidad en Educación Superior forman parte de un proceso complejo y dinámico de gobernanza epistémica que se ha ido configurando en el marco del estado evaluador (Elliot, 2002). No nos detendremos en los desarrollos de las políticas de calidad. Un examen de los mismos en Europa Occidental y con una perspectiva transnacional se ha llevado a cabo en diferentes contribuciones (véase, entre otros, Neave, 1995; Lorenzo Quiles y Cruz de Gracia, 2015). Lo cierto es que el término calidad resulta en la actualidad incuestionable (¿quién osa oponerse a la calidad?) y sin duda es un reflejo muy claro del giro lingüístico en sociología que Richard Harvey Brown sintetiza como: «el proceso de construir conocimiento sociológico - desde cualquier perspectiva teórica- que tiene mucho en común con los procesos con los que la gente común junta realidad y significado en sus vidas cotidianas». (Brown, 1983: 129). En efecto, «la noción de calidad ha sido socializada hasta tal punto entre el común de los ciudadanos-consumidores que se acepta su existencia (en términos ontológicos: se esencializa) y se constituye en criterio universal en la percepción y elección (...) de bienes y servicios». (Aguilar, 2006: 16-17). En el ámbito educativo su uso es tan frecuente como difícil -por no decir imposible-su definición. Todos hablamos de calidad dando por supuesto que sabemos de lo que hablamos aunque no sepamos definir el objeto o la cualidad a la que nos referimos. Forma parte de aquellos términos que son resultado del triunfo de la ambigüedad o cuya textura es tan abierta y maleable que acaban convirtiéndose en etiquetas, mantras o entelequias metafísicas (hurtadas de cualquier contenido político o histórico), al servicio de consensos amplios y fáciles. En palabras del sociólogo de la educación Carlos Lerena, «la cuestión radical no consiste en preguntarse o saber qué es lo que quiere decir la expresión calidad de la enseñanza; se trata, no de saber eso, sino precisamente de saber qué es lo que con esa expresión se quiere callar, esto es, qué se quiere evitar decir o qué se quiere ocultar». (Lerena, 1989:95). Estaríamos, entonces, ante una metáfora fundamental («root metaphor»), o lo que es lo mismo, ante una palabra a través de la cual percibimos el mundo social (Alasuutari y Qadir, 2016:637-638).

En nuestro lenguaje ordinario, no decimos que hacemos calidad educativa, sino que tenemos calidad educativa, como una propiedad que nos pertenece y no como un proceso del que participamos. Y con ello nos referimos a un producto (mensurable, contable) cuyo valor reside en el quantum o cantidad que expresa, más que en la experiencia a la que podría remitir. Cuando la calidad, así percibida y cristalizada, se naturaliza y se sustantiviza como un objeto de mero cálculo, y no se adjetiva como experiencia relevante, se está contribuyendo a la construcción de una suerte de marco cultural, de una gobernanza epistémica sustentada en una supuesta «objetividad tecnocrática» (Innerarity, 2016:11). En este sentido, la propuesta de los rankings presenta un primer gran inconveniente objeto de debates y críticas que se basa en pretender que, a través de unos índices, o indicadores, se capte una realidad multidimensional y que, a pesar de la cautela que se debería tener ante ellos, arrastren la capacidad performativa creando una suerte de nueva realidad que empuja a muchas universidades a planificar el modo de mejorar su puntuación en ellos.

Esta inversión de la calidad entendida como cantidad obedece a una confusión epistemológica sobre la que se ha erigido una poderosa industria académica. Es la confusión, que ya denunció el físico Georgescu-Roegen (1971), derivada de no distinguir entre conceptos aritmomórficos y conceptos dialécticos, ambos tan inevitables como necesarios para las ciencias sociales. Los primeros, aquellos que tienen forma aritmética, se pueden hacer corresponder con la serie de los números naturales o reales. Así, si queremos calcular la «longitud» de una mesa, la mediremos utilizando números. Pero hay conceptos que no son susceptibles de medición con números, que no son representables numéricamente, son dialécticos, 
y escapan a las leyes de la lógica formal. ¿Cómo podemos representar numéricamente la belleza, la vida o la muerte? Del mismo modo, ¿cómo podemos representar numéricamente la calidad? En realidad, la factoría de los rankings padece la enfermedad de la aritmomanía, una nueva versión del clásico positivismo de las ciencias, que invalida como no científico cuanto escapa a la medición aritmomórfica, a aquello que no es susceptible de un registro numérico. Los datos numéricos -inevitables y necesarios- no admiten discusión. Ahora bien, las nociones dialécticas - sin las cuales no existiría el lenguaje en el que se basa el conocimiento y la ciencia- no tendrían ningún sentido sin la discusión ni el debate del que se nutren y que generan. La pretensión de medir o calcular (una actividad aritmomórfica) la calidad educativa (una noción dialéctica) -como si esta tuviera peso, longitud o amplitud- es una contradicción en los términos. Sin embargo, buena parte de la literatura académica y del negocio sobre la calidad de la educación se basa en este oxímoron. No existe la calidad pura, ni la manera de medirla, pues el significado de este término depende del uso que hagamos del mismo en contextos determinados, bajo condiciones específicas y para intereses preestablecidos.

\section{Sobre los rankings}

Una vez establecido el marco discursivo, describimos y analizamos cuatro de los rankings más conocidos, tres de ellos de la League table-QS, ARWU y THE-, y otro basado en los resultados de investigación, el SCImago Institutions Ranking. Este último, determinado por sus autores no como un ranking sino como una ordenación de resultados.

Metodológicamente se diferencian en que algunos se sustentan en análisis cuantitativo de la producción científica, otros se basan en informaciones subjetivas sobre el prestigio y reputación y otros combinan ambos métodos. Los rankings sintéticos convierten los datos de cada indicador en puntuaciones y a cada una de ellos se les asigna un peso determinado en función de lo que consideran más importante los autores del ranking o del concepto de calidad subyacente. La ordenación dependerá de las puntuaciones obtenidas y las mejores universidades serán las que más cerca estén de los máximos valores.

El IREG (Group International Ranking Expert) movimiento académico vinculado favorablemente con la elaboración de los rankings (Barsky, 2014:64) se estableció en 2004 en el marco de la Reunión de Seguimiento de la Mesa Redonda sobre «Indicadores Estadísticos para Evaluación de la Calidad de Instituciones de Educación Superior: Metodologías de Ranking y Tablas de Posiciones», convocada conjuntamente por el Centro Europeo de la UNESCO para la Enseñanza Superior (CEPES) y el Instituto de Políticas de Educación Superior (IHEP) y elaboraron el documento titulado «Principios de Berlín sobre los Rankings de Instituciones de Educación Superion» que quiere servir de guía para productores y consumidores de rankings.

En dicho documento se describe la orientación a la filosofía de mercado de los rankings, destacando que estos no deben ser el único método de evaluación de los sistemas de Educación Superior. No obstante, tanto los rankings como los benchmarking pretenden medir la calidad de servicios, programas, proyectos, productos, organizaciones e instituciones forzando la comparación entre diferentes instituciones y sistemas y la competición entre ellos (Krüger y Molas, 2010).

Los usuarios de los rankings, según el IREG, pueden esperar y deben exigir que los rankings, como los Principios de Berlín indican, sean claros acerca de sus objetivos y grupos de destinatarios, reconozcan la diversidad de misiones institucionales, identifiquen las fuentes de información utilizadas y especifiquen los contextos culturales e históricos, teniendo en cuenta que existen diferentes valores y creencias acerca de 
lo que constituye la «calidad» en la educación superior. En el informe se afirma que los usuarios deben ser conscientes de que existen rankings diferentes con propósitos diferentes, que se dirigen a grupos diversos, seleccionan indicadores distintos y utilizan metodologías diferentes (IREG, 2015). Como señala Barsky (2014:65), parafraseando una irónica cita de Stella y Woodhouse (2006), parece como si una alerta sobre el peligro de fumar fuera convertida en un documento que dijera: «no hay problemas con fumar siempre y cuando usted evite los peligros del cigarrillo».

Retomaremos estos aspectos al final del texto, e iniciamos el recorrido siguiente de presentación de los rankings de un modo descriptivo y crítico buscando un debate interdisciplinar en un área copada por la Bibliometría y la Cientometría que codifican como verdades reveladas (Barsky, 2014) ideas que disimulan las dificultades históricas y limitaciones conceptuales con que se han construido los sistemas de evaluación de la calidad de las universidades y de las publicaciones científicas.

\section{a) Academic Ranking of World Universities (ARWU)}

El Ranking Académico de las Universidades del Mundo (Academic Ranking of World Universities - ARWU) fue publicado por primera vez en junio de 2003 por el Centro de las Universidades de Clase Mundial (Center for World-Class Universities - CWCU) de la Escuela Superior de Educación de la Universidad Jiao Tongde Shanghái de China, y su periodicidad es anual. Aunque el ARWU se creó con el objetivo de conocer el posicionamiento de las universidades de educación superior en China en comparación con las universidades de clase mundial, es el ranking anual más utilizado por universidades de investigación en todo el mundo.

Como afirma Barsky (2014) su creación estuvo estrechamente asociada a la decisión del sistema universitario chino, centralizado a nivel estatal y con el objetivo de tener un modelo a seguir para el avance de la formación de científicos en China siguiendo como modelo ideal el vigente en los países capitalistas más avanzados.

Se trata de una lista de universidades en que se clasifican unas 500, que representan el $2 \%$ del total mundial. Siguiendo las aportaciones de Barsky (2014), aparecen 149 universidades norteamericanas, con Harvard como el máximo referente, con 17 sobre las 19 primeras, seguidas de las británicas Oxford y Cambridge. Las universidades chinas aparecen a partir de la posición 151, lo que evidencia la importante diferencia entre el nivel de la calidad atribuido a estas universidades y el modelo ideal deseado.

ARWU publica tres rankings de carácter internacional: 1) ranking general para el que utiliza seis indicadores, 2) el ranking segregado por áreas de conocimiento y 3) el ranking por disciplinas específicas para los que se utilizan cinco indicadores, cuatro de ellos iguales que en el ranking general.

Para el ranking mundial de 500 universidades (ARWU) se utilizan indicadores relacionados con la calidad docente, basados en el número de alumnos y profesores que han ganado premios Nobel y medallas Fields. La productividad científica se mide a través de los números de investigadores altamente citados, de artículos publicados en revistas de Nature y Science, y de artículos indexados en Science Citation IndexExpanded (SCIE) y Social Sciences Citation Index (SSCI). Se tiene en cuenta también el número de artículos publicados en el Science Citation Index-Expanded (SCIE) y el Social Science Citation Index (SSCI) durante el año anterior y el rendimiento per cápita respecto al tamaño de una institución. Califica más de 1.200 universidades al año y las 500 mejores se publican en la web, a las 100 primeras se les asigna una posición individualizada y a las restantes una posición global. 
Para el ranking mundial de 200 universidades desagregadas por áreas de conocimiento (ARWUFIELD) se asigna un puesto individual para las 50 primeras universidades y el resto se agrupan. Se valoran cinco áreas de conocimiento: 1) Ciencias naturales y matemáticas; 2) Ingeniería, Tecnología e Informática; 3) Ciencias de la vida y agricultura; 4) Medicina clínica y Farmacia y 5) Ciencias Sociales.

Para el ranking mundial de 200 universidades desagregadas por disciplinas (ARWU-SUBJECT) igual que el anterior, se asigna un puesto individual para las 50 primeras universidades y el resto se agrupan. Las disciplinas analizadas son: 1) Matemáticas, 2) Física, 3) Química, 4) Informática y 5) Economía y empresa.

ARWU es el único ranking de carácter internacional que depende exclusivamente de datos obtenidos de forma independiente de las instituciones objeto de estudio y que no utiliza encuestas de opinión. Su diseño es exclusivamente investigador y es muy valorado por la calidad de sus datos y precisión y confianza de sus herramientas de análisis (Docampo, 2008).

Pero, como afirman Santos, Teodoro y Da Costa (2016) la principal crítica se centra en que todos los indicadores se destinan a una sola de las misiones de la universidad, la investigación. Como afirman estos autores «pensar que el número de premios Nobel o medallas Fields pueden ser un indicador de calidad de una universidad es seguramente una ficción». (2016:38). Continúan afirmando que la utilización exclusiva de bases de datos como Thomas Reuters, básicamente de lengua inglesa, desvalorizan las ciencias sociales y humanas.

Tabla I. Descriptor del ranking - ARWU

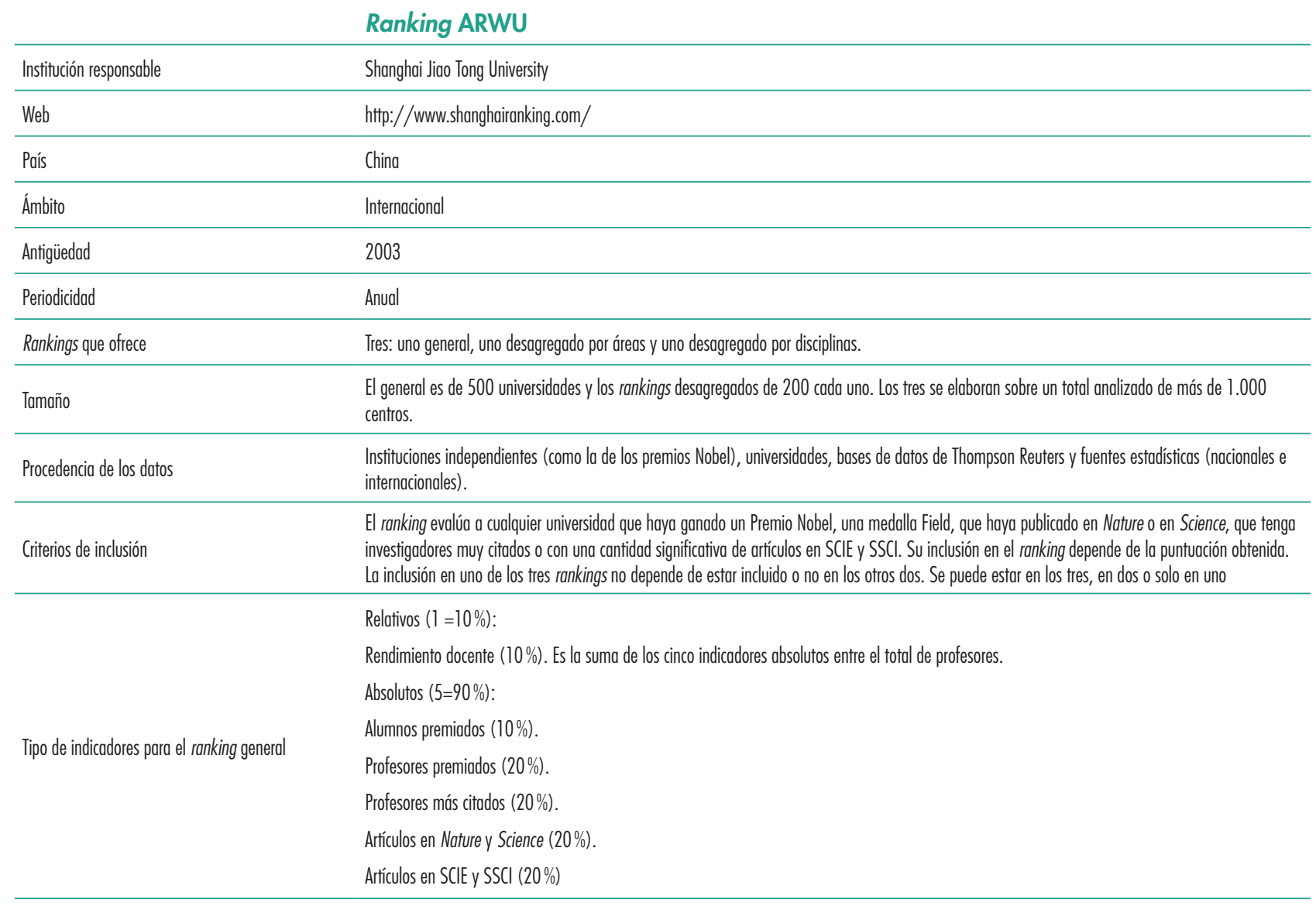




\begin{tabular}{ll}
\hline & Absolutos (5=100\%): \\
& Alumnos premiados (10\%). \\
Tipo de indicadores para el por áreas y disciplinas & Profesores premiados (15\%). \\
& Profesores más citados (25\%). \\
& Aríículos en SCIE y SSCI (25\%). \\
& Aríículos publicados en las revistas mejor valoradas respecto del total de revistas en cada campo (25\%)
\end{tabular}

Fuente: Adaptado de FICUE (2014:24).

\section{b) Times Higher Education World University Rankings (THE)}

El Times Higher Education World University Rankings (realizado por la revista Times Higher Education) proporciona información relacionada con la docencia, la internacionalización, la innovación, la investigación y la repercusión científica de las universidades a través de un ranking general y otro desagregado por áreas de conocimiento a partir de 13 indicadores. Asimismo, ofrece un ranking de universidades de menos de 50 años de antigüedad.

El ranking THE recoge la información a través del análisis bibliométrico de publicaciones y citas en los últimos diez años dentro de cada una de las seis áreas temáticas (Artes y Humanidades, Ciencias clínicas, Ingeniería y tecnología, ciencias biológicas, ciencias físicas y ciencias sociales), y una encuesta on line a académicos de todo el mundo realizada por Thomson Reuters.

Como señala la Guía de buenas prácticas para la participación de las universidades españolas en los rankings internacionales elaborada por el Ministerio de Educación y Deporte y coordinado por Sanz-Casado (2015:24), THE excluye a las instituciones que no tienen estudiantes de grado, las que enseñan una sola disciplina, o aquellas cuya producción en investigación fue menor 200 artículos al año. El ranking generalista de THE tiene dos grandes diferencias con ARWU. La primera es que un tercio de la ponderación del ranking se basa en una encuesta realizada a más de 10.000 académicos. El empleo de este tipo de indicadores subjetivos ha sido objeto de fuertes críticas, ya que, entraña deficiencias por su subjetividad y por la composición territorial de los expertos consultados ya que es imposible que un evaluador conozca todas las universidades del mundo para emitir un juicio (Pérez y Esparrells, 2009). Por otra parte, los datos los obtienen de las propias universidades y no de fuentes externas, con el problema de homogeneización entre universidades y países a la hora de su correcto empleo (SanzCasado, 2015).

Tabla II. Descriptor del ranking - THE

\begin{tabular}{ll} 
& Ranking THE \\
\hline Institución responsable & Revista Times Higher Education \\
\hline Web & httr://www.timeshighereducation.co.uk/worlduniversity-rankings/ \\
\hline País & Reino Unido \\
\hline Ámbito & Internacional \\
\hline Antigüedad & 2010 \\
\hline Periodicidad & Anual \\
\hline Rankings que ofrece & Dos: uno general y otro desagregado por áreas de conocimiento \\
\hline Tamaño & El general contiene 400 universidades. El ranking por áreas, 50 \\
\hline Procedencia de los datos & Las propias universidades, Thomson Reuters y la opinión de académicos de todo el mundo \\
\hline
\end{tabular}




\begin{tabular}{|c|c|}
\hline Criterios de inclusión & $\begin{array}{l}\text { Todas las universidades son analizadas de manera automática, exceptuando aquellas que no ofrezcan formación de grado, que enseñen en un campo } \\
\text { excesivamente especializado o hayan publicado menos de } 200 \text { por año. Su inclusión en los rankings depende de su puntuación }\end{array}$ \\
\hline \multirow{15}{*}{ Tipo de indicadores para el ranking general } & Relativo respecto el tamaño de cada universidad $(10=37 \%)$ : \\
\hline & Alumno/profesor. \\
\hline & Doctorados/licenciados o graduados. \\
\hline & Doctorados/profesores. \\
\hline & Ingresos totales / profesores. \\
\hline & Ingresos por investigación/profesores. \\
\hline & Artículos publicados por profesor/total profesores. \\
\hline & Estudiantes extranjeros/nacionales. \\
\hline & Docentes extranjeros/nacionales. \\
\hline & Publicaciones con un coautor extranjero/ total de publicaciones de la universidad. - Ingresos de empresas/profesores. \\
\hline & Absolutos respecto el tamaño de cada universidad $(1=30 \%)$ : \\
\hline & Número de citas que generan los artículos publicados por una universidad. \\
\hline & Subjetivos (sondeo de opinión) (2=33\%):- \\
\hline & Prestigio adjudicado a la docencia del centro. \\
\hline & Prestigio adjudicado a la investigación del centro \\
\hline
\end{tabular}

Fuente: Adaptado de FICUE (2014:42).

\section{c) QS World University Rankings®}

La organización británica QS World University Rankings (ranking QS) comenzó a publicar su propio comparativo de las mejores universidades a nivel internacional en 2009 a raíz del fin de su sociedad comercial con la revista Times Higher Education con la cual elaboró conjuntamente el ranking THE entre 2004 y $2009^{3}$.

Utiliza tres tipos de datos según el informe FICUE (2014): Encuestas on line entre académicos referidas al prestigio de las instituciones, las bases de datos $S$ copus ${ }^{4}$ para la información sobre la producción científica, ratios de recursos docentes e internacionalización extraída de las propias universidades para tratar la calidad educativa.

Desde su creación, el QS World University Ranking identifica más de 52 materias, pero solo se incluyen en el ranking aquellas que cumplen tres requisitos: recibir más de 20 respuestas entre académicos en las encuestas on line, que lleven más de cinco años con publicaciones en ese campo, y que la disciplina impartida se ofrezca en formación de grado y en programas de posgrado (FICUE, 2014).

Ofrece cuatro tipos de rankings:

1) El general, construido a partir de seis indicadores (reputación académica, reputación para las empresas, ratio de profesores por alumno, número de citas, internacionalización del profesorado e internacionalización del alumnado).

2) El de las mejores universidades de menos de 50 años, elaborado con los resultados del ranking general.

\footnotetext{
3 Este ranking se difunde directamente como QS a través de U.S. News \& World Report de los Estados Unidos. Hay que señalar que la ruptura mencionada con el Times no fue por cierto amigable y ambos grupos se critican duramente en sus documentos y compiten fuertemente por sus posiciones en el mercado de evaluación (Barsky, 2014).

4 Scopus es del grupo editorial Elsevier, creado en el año 2004, mientras que The Times se asoció con Thompson Reuters, lo que muestra la importante batalla económica por el monopolio del suministro de la información que está detrás de estos procesos supuestamente académicos.
} 
3) El desagregado por áreas de conocimiento (Artes y Humanidades, Ingeniería y Tecnología, Ciencias biológicas y medicina, Ciencias naturales y Ciencias sociales y gestión empresarial), a partir de cuatro indicadores.

4) El desagregado por disciplinas específicas, con los mismos indicadores que el anterior.

\section{Tabla III. Descriptor del QS World University Rankings}

\begin{tabular}{|c|c|}
\hline & Ranking QS \\
\hline Institución responsable & Quacquarelli Symonds (QS) \\
\hline Web & https://www.topuniversities.com/university-rankings \\
\hline País & Reino Unido \\
\hline Ámbito & Internacional \\
\hline Antigüedad & 2011 \\
\hline Periodicidad & Anual \\
\hline Rankings que ofrece & Tres: uno general, uno desagregado por áreas y uno desagregado por disciplinas \\
\hline Tamaño & $\begin{array}{l}\text { El general es de } 800 \text { universidades; el desagregado por áreas de } 400 \text {; y el de disciplinas de } 200 \text {. Los tres se elaboran sobre un total analizado de unas } \\
2.000 \text { instituciones }\end{array}$ \\
\hline Procedencia de los datos & Dos encuestas, base de datos Scopus, universidades y otras instituciones educativas de cada país. \\
\hline Criterios de inclusión & Las universidades seleccionadas son analizadas de manera automática. Su inclusión en el ranking depende de su puntuación. \\
\hline \multirow{10}{*}{ Tipo de indicadores para el ranking general } & Objetivos: \\
\hline & Relativos (4=50\%): \\
\hline & - Profesores contratados/dlumno (20\%) \\
\hline & - Citas que recibe cada profesor/ total de profesores (20\%) \\
\hline & - Docentes extranjeros/nacionales (5\%) \\
\hline & - Estudiantes extranjeros/nacionales (5\%) \\
\hline & Absolutos (0) \\
\hline & Subjetivos (sondeos de opinión) (2=50\%): \\
\hline & - Reputación académica (40\%) \\
\hline & - Reputación para empresas contratantes (10\%) \\
\hline
\end{tabular}

Fuente: Adaptado de FICUE (2014:8).

\section{d) SClmago-Institutions Rankings (SIR)}

SIR desarrollado por el grupo de investigación SCImago $L A B^{5}$, es un recurso para la evaluación de universidades y de instituciones de investigación científica de todo el mundo, que genera información de rankings con el fin de analizar los resultados de investigación. SCImago incluye una selección de indicadores bibliométricos, una cobertura inmensa de revistas de impacto y concentra sus intereses en países iberoamericanos (Lorenz y Cruz, 2015:168). SIR incluye y clasifica instituciones académicas que realizan investigación, ordenadas por un indicador compuesto que combina tres grupos de indicadores basados en: (1) investigación, (2) innovación e (3) impacto social medida por la visibilidad de su web. Como señalan sus autores, no se trata propiamente de un ranking si no de una ordenación a partir de la producción científica de las instituciones que debe entenderse como un rango predeterminado de distribución, no como una propuesta de ranking. El único objetivo de estos de informes es caracterizar los resultados de la investigación de la organización con el fin de proporcionar información útil para

5 Los miembros del grupo SCImago pertenecen al Consejo Superior de Investigaciones Científicas (CSIC) y a las Universidades de Granada, Alcalá de Henares y Carlos III de Madrid, Extremadura, Oporto (Portugal), Universidad Nacional de la Plata (Argentina) y Pontifica Universidad Católica de Valparaiso (Chile). 
las instituciones, los políticos y los gestores de oficinas de investigación, ya que es una herramienta válida capaz de analizar, evaluar y mejorar los resultados de investigación.

Sus parámetros de análisis son:

- Producción total de documentos publicados (tiene en cuenta la base de datos Scopus).

- Citas recibidas por publicación.

- Colaboración internacional (proporción de artículos publicados en cooperación con investigadores y centros extranjeros).

- Indicador SJR (SCImago Journal Rank) normalizado (media global de la relación entre el SJR de las revistas en que publica la misma institución y el SJR promedio de las revistas del área temática).

- Índice de citación normalizado (proporción entre el nivel de citas recibidas por la institución y el nivel medio de citas en las áreas temáticas de los artículos)

Las instituciones participantes se seleccionan con el único criterio de que tienen que ser instituciones universitarias y de investigación con más de 100 artículos publicados e incluidos en la base de datos Scopus. A efectos de la clasificación, el cálculo se genera cada año a partir de los resultados obtenidos durante un intervalo de cinco años cuyo último año corresponde a dos años antes de la edición del ranking. En la edición 2016 los resultados utilizados fueron los del período de cinco años (2010-2014). En la edición 2016 a nivel mundial se clasificaron en su totalidad a 5.147 instituciones, siendo 2.894 las correspondientes al sector de Educación Superior.

Tabla IV. Descriptor del Ranking - SIR

Ranking SIR

\begin{tabular}{|c|c|}
\hline & \\
\hline Institución responsable & SClmago Research Group \\
\hline Web & http://www.scimagoir.com/ \\
\hline País & España \\
\hline Ámbito & Internacional \\
\hline Antigüedad & 2009 \\
\hline Periodicidad & Anual \\
\hline Rankings que ofrece & Uno \\
\hline Tamaño & Analiza y publica datos de 3.290 instituciones (no solo universidades) \\
\hline Procedencia de los datos & Base de datos Scopus \\
\hline Criterios de inclusión & $\begin{array}{l}\text { Haber publicado al menos } 100 \text { documentos científicos de cualquier tipo (artículos, revisiones, cartas, conferencias, etc.) durante el último año del } \\
\text { periodo tomado en cuenta para realizar cada ranking anual }\end{array}$ \\
\hline \multirow{7}{*}{ Tipo de indicadores respecto tamaño de la universidad } & Absolutos (7): - \\
\hline & Artículos publicados \\
\hline & Artículos en colaboración con instituciones extranjeras \\
\hline & Artículos publicados en las revistas mejor valoradas \\
\hline & Promedio de citas recibidas/total de citas en el mundo \\
\hline & Grado de concentración o dispersión temática de la producción científica de la institución \\
\hline & Artículos más citados/total de artículos de un área. - Artículos en los que el autor principal pertenece a la institución evaluada \\
\hline
\end{tabular}

Fuente: Adaptación FICUE (2013). 


\section{Luces y sombras de los rankings}

A pesar de las críticas que puedan hacerse en contra de los rankings -muchas de ellas fundamentadas, entre otras cosas, por su cuestionable construcción metodológica-, no se puede negar su legitimidad (Altbach, 2006) ni el impacto que tienen en el ámbito universitario. Posiblemente uno de los efectos más destacables sea la poderosa influencia que ejercen «en las decisiones de los estudiantes sobre la universidad a la que deberían asistir, y cuanto estarían dispuestos a pagar» por un título de determinada institución (Ordorika y Lloyd, 2013:210).

De acuerdo con Marmolejo (2010), sin importar lo que pensemos, los rankings se han convertido en parte del entorno institucional de la educación superior. Aunque es cierto que estos comparativos «pueden ser una herramienta útil que ayuda a guiar el mejoramiento institucional» en el nivel superior (Marmolejo, 2010:2), también lo es que deben analizarse con escepticismo y manejarse con cuidado, como suscribe Baty (2012), editor del ranking THE.

Si bien los rankings son un instrumento legítimo y útil en muchos aspectos, uno de los principales problemas estriba en la facilidad que tienen para simplificar la realidad y crear la impresión de que una universidad es mejor que otra, basándose en una serie de indicadores usados arbitrariamente para medir la calidad de las universidades (Cheol Shin et al., 2011; Erkkilä, 2013; Federkeil et al., 2012; Marope et al., 2013; Ordorika y Lloyd, 2013).

Los rankings internacionales, principalmente de la League table, comparten el fuerte impacto mediático, pero, también, importantes críticas sobre su construcción metodológica, entre ellas, el hecho de que en algunos solo se analicen determinados campos científicos, se consideren solo unos pocos indicadores, se estudie solo un grupo de instituciones del sistema universitario o se abuse de indicadores bibliométricos o asociados a la producción de publicaciones científicas mostrando solamente una dimensión de las actividades universitarias (Sanz-Casado et al., 2013). Como señalan Pérez-Esparrels et al., (2010) existen opiniones que clasifican los rankings como un «sin sentido» ya que más allá de las limitaciones que presentan pecan de ingenuidad (Harvey, 2008) al medir exclusivamente lo que es posible medir, no lo que es relevante medir (Stella y Woodhouse, 2006).

Otro aspecto a destacar es la poca diferenciación entre el tamaño de las universidades. Así, una universidad muy grande, pero con un nivel de resultados muy pobre es un problema mucho mayor que una universidad con ese mismo nivel pero más pequeña. Pérez y Aldás (2016) señalan que algunos de los rankings más citados son de volumen, puesto que la mayoría de variables con las que se construye proporcionan datos absolutos (número de premios nobel o medallas Fields), pero no relativos al tamaño de la universidad. Otros rankings, como el QS World Universities Ranking, utilizan variables normalizadas por tamaño.

Los actuales rankings no suelen contemplan la responsabilidad social de las universidades, y no contemplan la multidimensionalidad, ni suelen analizar las asociaciones entre los indicadores, así como la implicación de otros factores externos

En este sentido, diversos informes, entre el que destaca la Guia de buenas prácticas para la participación de las universidades españolas en los rankings internacionales, (Sanz-Casado, 2015) señala una serie de riesgos a tener presentes en relación a los rankings, entre los que hemos seleccionado algunos, propuesto otros y ampliado el contenido a modo de diálogo: 
Riesgo de confundir lo que se puede medir con la que es importante medir. El planteamiento sistémico y organizado de la información de las publicaciones (ISI, THOMPSON) mantienen una estrecha relación con los principales rankings ya que éstos basan sus resultados de investigación basándose en dichas bases de datos, descuidando otros aspectos irrenunciables en la actividad de las universidades como la docencia, la innovación o la responsabilidad social. En la medida en que no se utilicen datos sobre estas otras actividades no será lícito extrapolar los resultados de investigación a las demás actividades (SanzCasado, 2015:9). La precisión reclamada para la medición puede en realidad ocultar la importancia de lo que no se mide. Existe, pues, una tensión entre lo que es fácilmente mensurable, pero puede no ser significativo, y lo que es de gran importancia, pero no puede medirse (Unterhalter, 2016).

Riesgo de utilizar indicadores sintéticos poco robustos. La principal característica de los rankings es que simplifican una realidad multidimensional y justamente esta característica los hace muy sensibles a las variables utilizadas. A ello se une la dificultad de agregar todos estos indicadores en uno solo para construir un índice sintético o la dificultad de contemplar conjuntamente variables relativas a cantidad y calidad. La asignación de pesos a los indicadores, o el empleo de indicadores basados en reputación implican un determinado grado de subjetividad (Sanz-Casado, 2015:9). ARWU recurre al número de Premios Nobel y medallas Fields como indicador para estimar la calidad de la docencia y el profesorado que trabaja en las universidades. Puede cuestionarse, sugieren Federkeil et al., (2012), si este indicador en realidad mide la excelencia en la investigación o mide el prestigio de las universidades para la atracción de talento.

Rankings como el THE o el QS utilizan algunos indicadores que los diferencian del ARWU, pues se valen de encuestas de opinión (conocidas como reputation surveys). Las encuestas utilizadas como herramientas para estimar la calidad de las universidades únicamente pueden ser relevantes si las personas que participan en la muestra tienen una opinión fundamentada en hechos sobre los que están evaluando, aspecto seriamente cuestionado (Flores y Villaseñor, 2015).

Riesgo de fijarse solo en la élite. Los rankings son elitistas por naturaleza: más de 16.000 universidades en el mundo nunca podrán clasificarse en alguno de estos comparativos (Rauhvargers, 2011). Los rankings internacionales se centran en un limitado número de universidades -las 500 que contempla el Ranking de Shanghai representan menos del 3\% de las existentes en el mundo- utilizando criterios inaplicables para evaluar a la gran mayoría de las instituciones que conforman los sistemas universitarios de cualquier país, incluidos aquellos con sistemas universitarios más potentes (Sanz-Casado, 2015:10). Por analogía, la utilidad los rankings para los sistemas universitarios requiere considerarlos desde una perspectiva más amplia e incluyente que la utilizada por los rankings internacionales. Esto sucede, como afirman Sanz-Casado et al., (2013:3), porque muchos rankings toman como modelos de universidad las de Estados Unidos (o de los países anglosajones) en los que predomina un tipo de universidad más elitista y orientada fundamentalmente a la investigación. Este modelo impide detectar la diversidad de tipologías que existen en otros contextos y que resultan igualmente valiosas para el desarrollo integral de su entorno.

Riesgo de comparar universidades heterogéneas. Las universidades poseen distintas especializaciones por campos científicos o en sus actividades (más o menos orientadas a la docencia, a la investigación, o al desarrollo tecnológico). Las universidades también son heterogéneas en cuanto a su historia, coexistiendo universidades varias veces centenarias con universidades de reciente creación (Sanz-Casado, 2015:10). Marginson (2006) llama la atención sobre que los rankings globales usan de esta forma sobreentendida 
una distinción discriminatoria entre dos tipos ideales de universidades, la universidad de investigación intensiva y la de educación vocacional, masificada. Por su propia naturaleza institucional compleja, las universidades, que agrupan carreras disímiles que integran disciplinas con tradiciones diferenciadas en relación a los campos del conocimiento y de las aproximaciones epistemológicas a estos, parecen no poder ser sometidas a un tratamiento similar a escala planetaria. No todas las universidades tienen los mismos objetivos ni la misma historia institucional y nacional, por lo que es cuestionable la consideración de las «mejores universidades».

Riesgo de confundir las partes con el todo: distribución territorial. Tomar en consideración el número de veces que son citadas las publicaciones de los investigadores como indicador de calidad representa otro problema, ya que los científicos norteamericanos que son los más numerosos tienden a citar a sus compatriotas (Flores y Villaseñor, 2015), dejando de lado especialmente aquellos que no son anglófonos. El inglés es el idioma de las ciencias duras, pero no necesariamente es el idioma principal en campos como las humanidades y las ciencias sociales, donde los investigadores hispanos son más representativos (Altbach, 2006). Esta cuestión representa otra de las características de los rankings que perjudica, por una parte, a las universidades de los países en donde el inglés no es la lengua oficial y por otra a determinadas disciplinas como las Ciencias Sociales. Acerca de esta notable deficiencia metodológica, Federkeil et al., (2012: 36) señalan que «la reputación de las universidades como atributo de su calidad [...] se ve fuertemente afectado por la estructura de la muestra en términos de su distribución regional».

Riesgo de no comprender la dimensión de negocio de los rankings. Siguiendo a Barsky (2014) podemos afirmar que los mercados globales incluyen también el gran negocio de la evaluación universitaria: los rankings. A partir de 2007 los rankings fueron compilados por la consultora de temas educacionales Quacquarelli- Symonds (QS), dirigida por Nunzio Quacquarelli. En el año 2009 Times Higher Education cortó sus vínculos con QS y firmó un acuerdo con Thomson Reuters.

Thomson Reuters, nacida en 2008, es una de las grandes compañías que suministra información para empresas y profesionales sobre diversos temas (Barsky, 2014:37) y principalmente es el mayor proveedor de información financiera del mundo. Su plataforma proporciona a los académicos y administradores universitarios acceso a bases de datos del mundo de 12.000 de las revistas de mayor impacto académico y más de 110.000 resúmenes de congresos. Abarca las Ciencias Exactas y Naturales, las Ciencias Sociales, Artes y Humanidades. Tiene su sede en Nueva York, opera en más de 100 países y emplea unas 50.000 personas (Barsky 2014:38). Esta alianza entre una poderosa compañía y la medida de la calidad de las universidades debería hacernos pensar en las reglas del juego con una orientación comercial que acaban asumiéndose como imperativos irrenunciables y forman un entramado que trasciende cualquier concepción de calidad.

\section{Consideraciones finales}

La llamada «cultura de la evaluación» (Elliot, 2002), desplegada en el campo de la educación superior, ha requerido y propiciado el desarrollo de diversos métodos e instrumentos para medir, calificar y dar seguimiento al desempeño y resultados de las funciones académicas y actividades de gestión de las instituciones. Dentro de la gama de modalidades evaluativas (Brooks, 2005), concretamente la evaluación comparativa, ha adquirido relevancia a medida que ofrece referentes para contrastar los logros y avances de instituciones ante los resultados obtenidos por otras unidades del conjunto. En tal contexto, los rankings y tablas de posiciones (league tables), cuya elaboración se remonta varias décadas atrás en el ámbito anglosajón a nivel nacional, se ha expandido internacionalmente. 
Si captamos con cierta distancia crítica el conjunto descrito en el texto, podemos apreciar una serie de características en las que parece no importar tanto el significado como el significante. En la actualidad, en efecto, asistimos a la configuración de una neoescolástica, en la que de algún modo se reedita el debate entre realistas y nominalistas (el nombre de la calidad no es la calidad), y en la que prevalece más el cumplimiento de una serie de requisitos formales que de cuestiones sustantivas. En segundo lugar, en los mecanismos de aseguramiento de la calidad se aprecia un énfasis en los resultados (concebidos como productos), antes que en los procesos educativos. Paradójicamente, las exigencias de calidad están medidas o mediadas por instrumentos y procedimientos hegemónicos principalmente de carácter cuantitativo, que acaban cristalizando en una poderosa industria contable (indicadores, rankings, comparaciones...). Esta manera de medir la educación desplaza las lógicas institucionales hacia nuevas lógicas que subordinan el conocimiento a lo que se ha dado en llamar economía del conocimiento. Por otra parte, llama la atención que, además de los instrumentos al servicio del crecimiento productivo, no se hayan desarrollado en igual medida instrumentos vinculados primordialmente al desarrollo social o de ciudadanía.

En el fondo, la consideración de la medida de la calidad educativa a través de los rankings, parte de un equívoco, a saber: medir significa registrar, informar, es una actividad descriptiva; evaluar significa emitir juicios de valor fundamentados, es una actividad normativa. Pero medir no es evaluar. No se pueden derivar consecuencias normativas - como de hecho se hace- de cuestiones descriptivas.

Lo que se desprende del marco que hemos presentado es la necesidad de un profundo cambio de perspectiva para recuperar y reconstruir los discursos sociales de los actores que participan y están implicados en las propias instituciones. Al fin y al cabo, el valor de la calidad educativa no depende tanto de la rendición de cuentas (accountability) sino de la rendición de sentido (sense-abilty). Por eso, los instrumentos que ayudan a medir la calidad educativa han de estar al servicio del valor educativo (es decir, de aquello que vale la pena), y no a la inversa: una calidad educativa subordinada a los instrumentos que la miden, ignorando el horizonte de los auténticos fines emancipadores que ha guiado y ha de seguir guiando el desarrollo de la educación superior.

En los rankings analizados en el presente texto se percibe el supuesto tácito de que una buena universidad es aquella cuya investigación es altamente competitiva sin medir otros parámetros. El prestigio en ese ámbito eclipsa otras virtudes educativas.

\section{Referencias bibliográficas}

Aguilar, Luis (2006). Todo sea por la calidad. Tramar el cambio en educación. Alzira: Germania.

Alasuutari, Pertti y Qadir, Ali (2016): "Imageries of the social world in epistemic governance". International Sociology, 31, 16-652.

Altbach, Philip (2006): “The Dilemmas of Ranking” en Philip Altbach (ed.): International higher education: Reflections on policy and practice, 77-80. USA: Chesnut Hill, www.bc.edu/content/dam/files/research_sites/cihe/pubs/Altbach_2006_Intl_HigherEd.pdf

Barsky, Osvaldo. (2014): "La evaluación de la calidad académica en debate: los rankings internacionales de las universidades y el rol de las revistas científicas". Buenos Aires: Ed. Teseo, Universidad Abierta Interamericana.

Baty, Phil (2009): "Rankings 09: Talking Points". The Times Higher Education, 8. 
Baty, Phil (2012): “Rankings don't tell the whole story - Handle them with care. University World News, 227.

Beltrán, José y Montané, Alejandra (2015): "Higher Education in Spain: Framework for Governance and Autonomy in Spanish Universities" en K. M. Joshi y Saeed Paivandi (eds.): Global Higher Education: Issues in Governance. New Delhi: B. R. Publishing House.

Beltrán, José; Montané, Alejandra; Gabaldón-Estevan, Daniel (2016): "Higher Education in Spain: Framework for Equity" en Saeed Paivandi, Saeed y K. M. Joshi (eds.): Equity in Higher Education. A Global Perspective. New Delhi: Studera Press.

Brown, Richard Harvey (1983): "Theories of rethoric and the rethorics of theory: Toward a political phenomenology of sociological truth". Social Research, 50, 126-157.

Brooks, Rachelle L. (2005): "Measuring University Quality”. Review of Higher Education, 29 (1), 1-22.

Bruner, José Javier; Uribe, Daniel (2007). Mercados universitarios: el nuevo escenario de la educación superior. Chile: Ediciones Universidad Diego Portales.

Campbell, David F. y Carayannis, Elias, G. (2013). Epistemic Governance in Higher Education. New York: Springer.

Docampo, Domingo (2008): "Rankings internacionales y calidad de los sistemas universitarios". Revista de Educación, número extraordinario, 149-176.

Elliot, John (2002): “La reforma educativa en el Estado evaluador”. Perspectivas, XXXII, 3.

Erkkilä, Tero (2013). Global University Rankings: Challenges for European Higher Education. New York: Palgrave Macmillan.

FICUE (2014): Fotografía Internacional de la Calidad de las Universidades Españolas (2014) Estudio de la posición de las universidades españolas en algunos rankings internacionales. Fundación para la Proyección Internacional de las Universidades Españolas, Universidad.es www.universidad.es

FICUE (2013): Análisis de la posición de las universidades españolas en los rankings internacionales. Fundación para la Proyección Internacional de las Universidades Españolas, Universidad.es www. universidad.es. [Versión provisional].

Federkeil, Gero; Van Vught, Frans A. y Westerheijden, Don F. (2012). "An Evaluation and Critique of Current Rankings" en F. A. van Vught and F. Ziegele (eds.): Multidimensional Ranking. The Design and Development of U-Multirank. Netherlands: Springer Science+Business Media B.V.

Flores, Jorge Enrique y Villaseñor, Jorge Ignacio (2015): "Perspectivas actuales sobre los rankings mundiales de universidades". Revista de Educación Superior, 44 (175), 44-67.

Georgescu-Roegen, Nicholas (1971). The Entropy Law and the Economic Process. Cambridge: Harvard University Press.

Gómez, Tomás y Puente, Xavier (2012a): “El valor de los ranking universitarios (segunda parte)”. Bitácora de gestión universitaria. Disponible en: http://finanzasuniversitarias.edunomia.es/2012/02/26/ el-valor-de-los-rankings-universitarios-segunda-parte/

Gómez, Tomás y Puente, Xavier (2012b): “El valor de los rankings universitarios”. Economías Inteligentes.com. Disponible en: http:/ / economiasinteligentes.com/2012/04/22/el-valor-de-los-rankingsuniversitarios.html 
Harvey, Lee (ed.) (2008): "Ranking on Higher Education Institutions: a Critical Review". Quality in Higher Education, 14(3), 187-207.

Hazelkorn, Ellen (2015): Rankings and the Reshaping of Higher Education: the Battle for World-Class Excellence. London: Palgrave Macmillan.

Illich, Ivan (1974). La convivencialidad. Barcelona: Barral editores.

Innerarity, Daniel (2016): “La sociedad de los cálculos”. El País, 29 de octubre.

IREG - Observatory on Academic Ranking and Excellence (2015): Pautas para grupos de interés de rankings académicos. Disponible en: http://iregobservatory.org/en/pdfy/IREG-Guidelines_Spanish.pdf.

Krüger, Karsten y Molas, Alba (2010): "Rankings Mundiales de Universidades: Objetivos y Calidad". An@cne revista electrónica de recursos en Internet sobre Geografía y Ciencias Sociales, 129.

Lerena, Carlos (1989): "De la calidad de la enseñanza. Valor de conocimiento y valor político de una entelequia”. Política y sociedad, 3, 91-99.

Lindblad, Sverker (2008): "Navigating in the field of university positioning: on international rankings lists, quality indicators and higher education governing". European Educational Research Journal, 7 (4), 438-450.

Liu, Nian; Cheng, Ying y Liu, L. (2005): “The Academic ranking of world universities using scientometrics." Scientometrics, 64, 101-109.

López, Ana María; Pérez-Esparrells, Carmen; Montañez, Myriam (2008): "Una aproximación a un indicador sintético para la evaluación del sistema universitario público español”. Investigaciones de economía de la educación, 3, 105-112.

Lorenzo Quiles, Oswaldo y Cruz de Gracia, Efraín (2015): “Calidad y evaluación de la Educacion Superior. Una perspectiva transnacional a través de los ranking”. DEDiCA. Revista de Educação e Humanidades, 8, julio, 155- 174.

Lorenzo Quiles, Oswaldo y Cruz de Gracia, Efraín (2014): "Rankings de evaluación de las instituciones de educación superior. Un análisis comparativo”. Pensamiento y acción, 19, 62-73.

Marginson, Simon (2006): Global University Rankings at the end of 2006: Is this the hierarchy we have to have? OECD/IMHE \& Hochschulrenkonferenz, Germany: Workshop 4-5, Bonn. Institutional Diversity: Rankings and typologies in higher education. http://www.cshe.unimelb.edu.au/people/ marginson_docs/IMHE_Bonn_rankings041206.pdf

Marginson, Simon (2010). "National and International Rankings of Higher Education" en P. Peterson; E. Bakery and B. McGaw. International Enciclopedia of Education, $3^{\mathrm{a}}$ (ed.) Amsterdam: Academic Press,

Marginson, Simon y Van der Wende, Marij (2007): “To rank or to be ranked: The impact of global rankings in Higher Education". Journal of Studies in International Education, 11 (3-4), 306-329.

Marmolejo, Francisco (2010): “The Madness of Rankings”. The Chronicle of Higher Education. http://chronicle.com/blogs/worldwise/the-madness-of-rankings/27100.

Marope, Priscilla Toka Mmantsetsa; Wells, Peter J. y Hazelkorn, Ellen (eds.) (2013). Rankings and accountability in Higher Education: Uses and Misuses. UNESCO. 
Montané, Alejandra; Beltrán, José y Gabaldón-Estevan, Daniel (2017): “Higher Education in Spain. Framework for Quality Assurance" en Stamelos Georgios, K. M. Joshi y Saeed Paivandi (eds.): Quality Assurance in Higher Education. A Global Perspective. Delhi, India: Studera Press.

Neave, Guy (1995): “Las políticas de calidad: desarrollos en Enseñanza Superior en Europa Occidental”. Revista de Educación, 308, 7-29.

Orodorika, Imanol y Lloyd, Marion (2013): "A decade of international university rankings: a critical perspective from Latin America" en Priscilla TokaMmantsetsa Marope; Peter J. Wells y Ellen Hazelkorn (eds.): Rankings and accountability in Higher Education: uses and misuses. Paris: UNESCO Publishing. http:// unesdoc.unesco.org/images/0022/002207/220789e. pdf

Pérez, Francisco; Aldás, Joaquín (dirs.) (2016): "U-Ranking 2016. Indicadores sintéticos de las universidades españolas". Instituto Valenciano de Investigaciones Económicas, Fundación BBVA, marzo (cuarta edición).

Pérez-Esparrells, Carmen y López, Ana María (2009): "Los rankings de las instituciones de Educación Superior: Una revisión del panorama internacional”. Calidad en la educación. Disponible en: http:// www.cned.cl/public/secciones/seccionpublicaciones/doc/63/cse_articulo809.pdf

Pérez, Francisco. (dir.) (2014): Rankings ISSUE 2014. Indicadores sintéticos de las universidades españolas. Fundación BBVA e IVIE. http://www.fbbva.es/TLFU/dat/Informe_URanking_FBBVA_ Ivie_2016.pdf

Pulido, Antonio (2009). El futuro de la Universidad. Un tema para debate dentro y fuera de las Universidades. Madrid: Delta Publicaciones.

Rauhvargers, Andrejs (2011): “Global University Rankings and Their Impact. Brussels: European University Association”. www.eua.be/pubs/ Global_University_Rankings_and_Their_Impact.pdf

Santos, Eduardo; Teodoro, António y Da Costa Junior, Reinaldo (2016): "Rankings Universitários: entre a regulação do mercado e a difusao de modelos organizacionais - o caso brasileiro" en Angelo Del Vecchio y Eduardo Santos (orgs.): Educação Superior no Brasil; Modelos e Missoes Institucionais. Sao Paulo: BT Academica.

Sanz-Casado, Elías et al., (2013): "Rankings nacionales elaborados a partir de múltiples indicadores frente a los de índice sintético". Revista española de documentación científica, 36 (3).

Sanz-Casado, Elías. (coord.) (2015). Guía de buenas prácticas para la participación de las universidades españolas en los rankings internacionales. Madrid: Ministerio de Educación, Cultura y Deporte.

Shin, Jung Cheol; Toutkoushian, Robert K. y Teichler, Ulrich, (eds.) (2011). University Rankings. Theoretical Basis, Methodology and Impacts on Global Higher Education. Dordrecht: Springer.

Stella, Antony y Woodhouse, David (2006): "Ranking of Higher Education Institutions". Occasional Publications series, 6 .

Unterhalter, Elaine (2017): "Negative capability? Measuring the unmeasurable in education". Comparative Education, 53 (1), 1-16.

Wang, Qi; Cheng, Ying; Liu, Nian (2012). Building World-Class Universities Different Approaches to a Shared Goal. Rotterdam: Sense Publishers. 


\section{Notas biográficas}

Alejandra Montané López es Doctora en Pedagogía. Licenciada en Filosofía y Ciencias de la Educación. Profesora e investigadora del Departamento de Didáctica y Organización Educativa de la Universidad de Barcelona. Miembro de RIAIPE: Red Iberoamericana de Investigación en políticas educativas y del grupo de investigación consolidado Formación docente e innovación pedagógica (FODIP), iniciativas en las que ha desarrollado una amplia producción científica. Forma parte del grupo de CLACSO Universidades y Políticas de Educación Superior.

José Beltrán Llavador es Doctor en Filosofía. Profesor titular e investigador del Departamento de Sociología y Antropología Social de la Universidad de Valencia. Miembro de RIAIPE: Red Iberoamericana de Investigación en políticas educativas y del comité de investigación (CI 13) de Sociología de la Educación en la Federación Española de Sociología (FES), iniciativas en las que ha desarrollado una amplia producción científica. Forma parte del grupo de CLACSO Universidades y Políticas de Educación Superior.

António Teodoro es Doctor en Ciencias de la Educación por la Universidade Nova de Lisboa. Profesor Catedrático de la Universidade Lusófona de Humanidades e Tecnologias (ULHT), en Lisboa. Profesor de sociología de la educación y educación comparada. Director del Centro de Estudos Interdisciplinares em Educação e Desenvolvimento (CeiED), de la Universidade Lusófona. Vice-Presidente del Comité de Investigación de Sociología de la Educación (RC04) de la Asociación Internacional de Sociología (20062014). Cofundador del Instituto Paulo Freire de Portugal. Coordinador de la Red Ibero-Americana de Investigación en Políticas Educativas (RIAIPE). Fundador de la Sección de Educación Comparada de la Sociedad Portuguesa de Educación Comparada (SEC-SPCE). Coordinador del grupo CLACSO Universidades y Políticas de Educación Superior. 